\title{
NOD2 and inflammation: current insights
}

This article was published in the following Dove Press journal:

Journal of Inflammation Research

\author{
Anna Negroni' \\ Maria Pierdomenico ${ }^{2}$ \\ Salvatore Cucchiara ${ }^{2}$ \\ Laura Stronati ${ }^{3}$ \\ 'Division of Health Protection \\ Technologies, Territorial and \\ Production Systems Sustainability \\ Department, ENEA, Rome, Italy; \\ ${ }^{2}$ Department of Pediatrics and \\ Infantile Neuropsychiatry, Pediatric \\ Gastroenterology and Liver Unit, \\ Sapienza University of Rome, Rome, \\ Italy; ${ }^{3}$ Department of Cellular \\ Biotechnology and Hematology, \\ Sapienza University of Rome, Rome, \\ Italy
}

\begin{abstract}
The nucleotide-binding oligomerization domain (NOD) protein, NOD2, belonging to the intracellular NOD-like receptor family, detects conserved motifs in bacterial peptidoglycan and promotes their clearance through activation of a proinflammatory transcriptional program and other innate immune pathways, including autophagy and endoplasmic reticulum stress. An inactive form due to mutations or a constitutive high expression of NOD2 is associated with several inflammatory diseases, suggesting that balanced NOD2 signaling is critical for the maintenance of immune homeostasis. In this review, we discuss recent developments about the pathway and mechanisms of regulation of NOD2 and illustrate the principal functions of the gene, with particular emphasis on its central role in maintaining the equilibrium between intestinal microbiota and host immune responses to control inflammation. Furthermore, we survey recent studies illustrating the role of NOD2 in several inflammatory diseases, in particular, inflammatory bowel disease, of which it is the main susceptibility gene.
\end{abstract}

Keywords: innate immunity, intestinal homeostasis, ER stress, autophagy, inflammatory bowel disease, extraintestinal disease

\section{Introduction}

The human body is constantly in contact with a myriad of microorganisms, either pathogens or commensals. Innate immune system, which provides a first line of defense against many common microbes, is essential for an appropriate tissue homoeostasis as well as for common bacterial infections, and its dysfunction leads to infectious, inflammatory and autoimmune diseases.

Innate immune response relies on recognition of evolutionarily conserved structures on the microorganisms, termed pathogen-associated molecular patterns (PAMPs), through a limited number of germ line-encoded pattern recognition receptors (PRRs) present on the host cell surface or in the intracellular compartments. ${ }^{1}$ Among the latter, nucleotide-binding and oligomerization domain containing protein 2 (NOD2) is a cytosolic receptor belonging to the nucleotide-binding oligomerization (NOD)-like receptor (NLR) family. ${ }^{2}$ NOD2 is able to detect intracellular muramyl dipeptide (MDP), a component of the bacterial wall that is ubiquitously present in bacterial peptidoglycan. ${ }^{3}$ Upon activation by ligand, NOD2 mediates innate immune response triggering proinflammatory responses. NOD2 mutation or altered expression has been found in patients with chronic inflammatory disorders such as Crohn's disease (CD), Blau syndrome (BS) and early-onset sarcoidosis (EOS). ${ }^{4-9}$ In this review, we summarize the current knowledge about NOD2 functions and regulation, as well as its involvement in chronic inflammatory diseases.
Correspondence: Laura Stronati Department of Cellular Biotechnology and Hematology, Sapienza University of Roma, Viale Regina Elena 324, $0016 \mathrm{I}$ Rome, Italy

Tel +390649974393

Email laura.stronati@uniromal.it 


\section{The NLR family}

The 23 NLR family members, intracellular sensors of PAMPs, share a common organization consisting of a C-terminal leucine-rich repeat (LRR) domain with regulatory and ligand recognition functions, a central nucleotide-binding and oligomerization domain (NBD) and an N-terminal effector-binding domain. The type of effector domain results in the division of NLR proteins into five subfamilies: acidic transactivation domain (NLRA); baculovirus inhibitor repeat, BIR (NLRB); caspase recruitment domain, CARD (NLRC); pyrin domain (NLRP) and NLRX1 that localizes to the mitochondria and has no homology to any known $\mathrm{N}$-terminal domain (Figure 1). ${ }^{10,11}$ NOD2 belongs to the NLRC subfamily of NLRs and, after NOD1, has been the second member of the family to be identified. ${ }^{12}$ NOD2 receptor, encoded by the NOD2/CARD15 gene, mapping on chromosome 16q12.1 in humans, consists of 1040 amino acids and has a molecular weight of $110 \mathrm{kDa} .{ }^{12}$ It is expressed in monocytes, macrophages, dendritic cells, hepatocytes, preadipocytes, epithelial cells of oral cavity, lung and intestine, with higher expression in ileal Paneth cells and in intestinal stem cells. ${ }^{13,14}$ NOD2, like NOD1, is a cytoplasmic protein, although it is recruited in the plasmatic membrane where it detects bacterial invasion at the point of entry. ${ }^{15}$

\section{NOD2 signaling}

The innate immune system is critical for clearing infection and averting excessive tissue damage. NOD2, an intracellular receptor of microbial components derived from bacterial peptidoglycan, contributes to the maintenance of mucosal homeostasis and the induction of mucosal inflammation. Structurally, NOD2 protein is composed of two tandem $\mathrm{N}$-terminal CARDs that function as effector domain and mediate specific homophilic interaction with downstream CARD-containing molecules. ${ }^{16}$ On activation by MDP, a cell wall component of both Gram-positive and Gram-negative bacteria, through LRR domain, NOD2 undergoes selfoligomerization and recruitment of the downstream adaptor molecule, the kinase receptor interacting protein 2 (RIP2 also known as RICK, CARDIAK, CCK and Ripk2), via homophilic CARD-CARD interaction. ${ }^{17-19}$ Active RIP2 leads to ubiquitination of nuclear factor-kappa $\mathrm{B}(\mathrm{NF}-\mathrm{\kappa B})$ essential modulator, resulting in activation of IאB kinase (IKK) complex that phosphorylates NF- $\kappa B$ inhibitor- $\alpha(\operatorname{IKB} \alpha)$, the inhibitor of transcription factor NF- $\kappa \mathrm{B}$, which translocates to the nucleus and starts transcription of proinflammatory genes, including cytokines, growth factors and factors responsible for stimulation of immune cells. ${ }^{20,21}$ RIP2 targets transforming growth factor- $\beta$-activated kinase 1 , which, through IKK

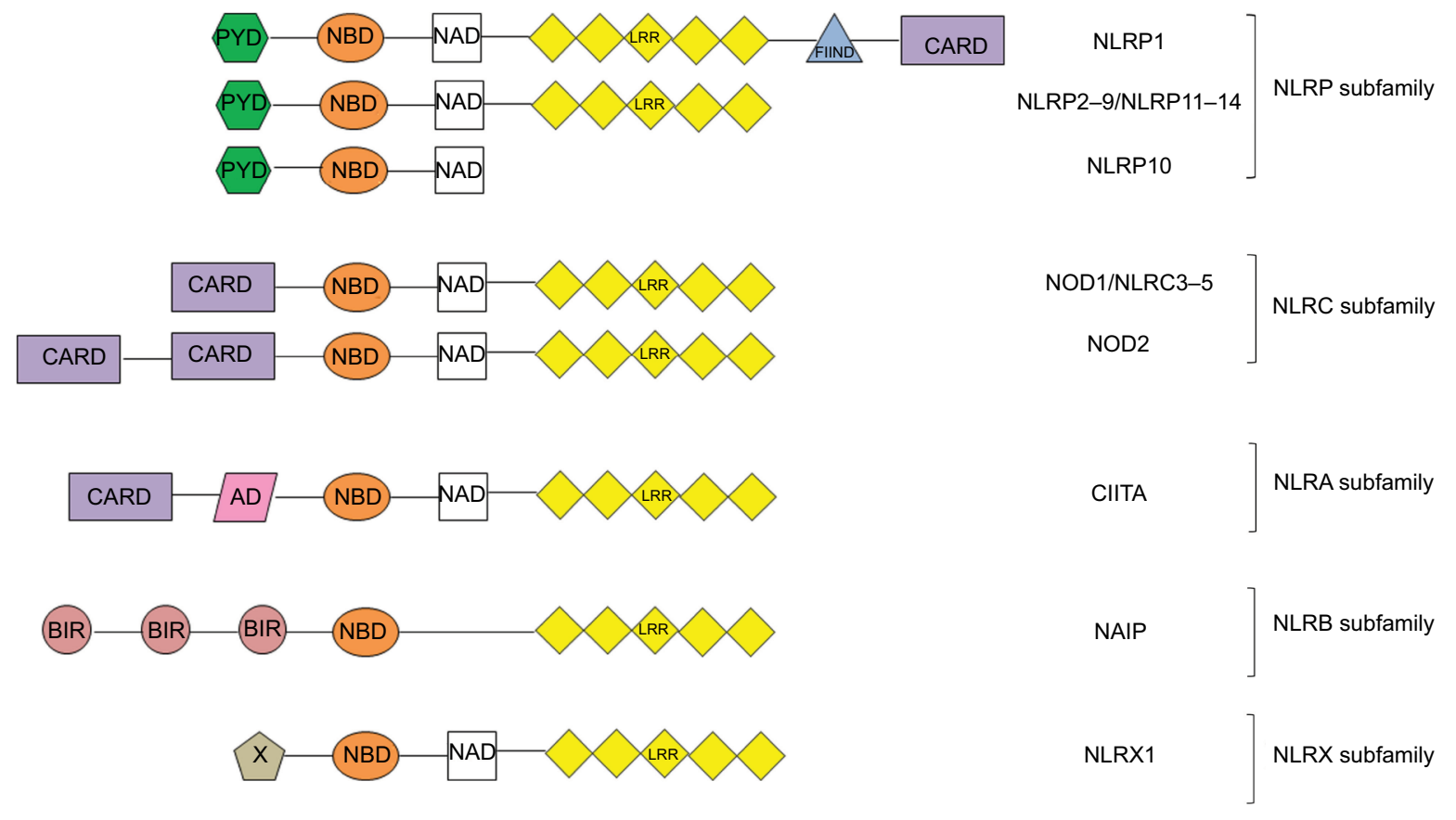

Figure I Structure of the NLR subfamilies.

Abbreviations: AD, atopic dermatitis; BIR, baculovirus inhibitor repeat; CARD, caspase recruitment domain; CIITA, class II major histocompatibility complex transactivator; FIIND, function to find domain; LRR, leucine-rich repeat; NAD, NBD-associated domain; NBD, nucleotide-binding domain; NLR, NOD-like receptor; NLRA, acidic transactivation domain; NLRB, baculovirus inhibitor repeat; NLRC, caspase recruitment domain; NLRP, NLR family pyrin domain; NOD, nucleotide-binding oligomerization domain; PYD, pyrin domain; $X$, unknown effector domain. 
complex, activates MAP kinases and transcription factor Activator Protein 1 involved in cell proliferation, differentiation and apoptosis. ${ }^{18,22}$ NOD2 is also known to bind and activate caspase-1, through its CARD domain, and starts interleukin (IL)- $1 \beta$ processing. ${ }^{23}$ Moreover, MDP challenge promotes the formation of NOD2-NLR family pyrin domain containing 1 complex that induces caspase-1-dependent IL- $1 \beta$ secretion in response to Bacillus anthracis or Yersinia pseudotuberculosis infection. ${ }^{23,24}$ Recently, NOD2 has been suggested to have a role in the autophagic process due to its interaction with the autophagy protein Autophagy Related 16 Like 1 (ATG16L1), which has been described below in-depth (Figure 2). ${ }^{25}$

\section{NOD2 regulation}

The complexity of NOD2 signaling is underlined by the evidence that many cellular proteins interact with NOD2 directly and regulate positively or negatively its functional activity. ${ }^{26}$ Among these, Erbin, Centaurin B1, Angio-Associated
Migratory Cell Protein, Carbamoyl-Phosphate Synthetase 2, Mitogen-Activated Protein Kinase Binding Protein 1 (JNKBP1) and heat shock protein (HSP) 90 have been shown to interact with NOD2 and regulate downstream signaling events. ${ }^{26-30}$ Recently, Suppressor of Cytokine Signaling-3 was found to recruit the ubiquitin machinery to NOD2, facilitating its proteasomal degradation. ${ }^{30}$ Interferon regulatory factor 4 (IRF4) is another negative regulator of NOD2-dependent $\mathrm{NF}-\kappa \mathrm{B}$ signaling through inhibition of RIP2 polyubiquitination in human dendritic cells. ${ }^{31}$ On the contrary, HSP70 and FERM and PDZ domain-containing protein 2 act as positive regulators of NOD2 signaling: the first one, after binding with NOD2, leads to increase in NF- $\kappa$ B activity and the second one favors NOD2 localization at the plasma membrane. ${ }^{29,32}$

Furthermore, important posttranslational modifications are required to control NOD2 signaling. ${ }^{33}$ The E3 ubiquitin ligases Pellino 3 and X-linked inhibitor of apoptosis protein, by ubiquitinylating RIP2, are important mediators in the

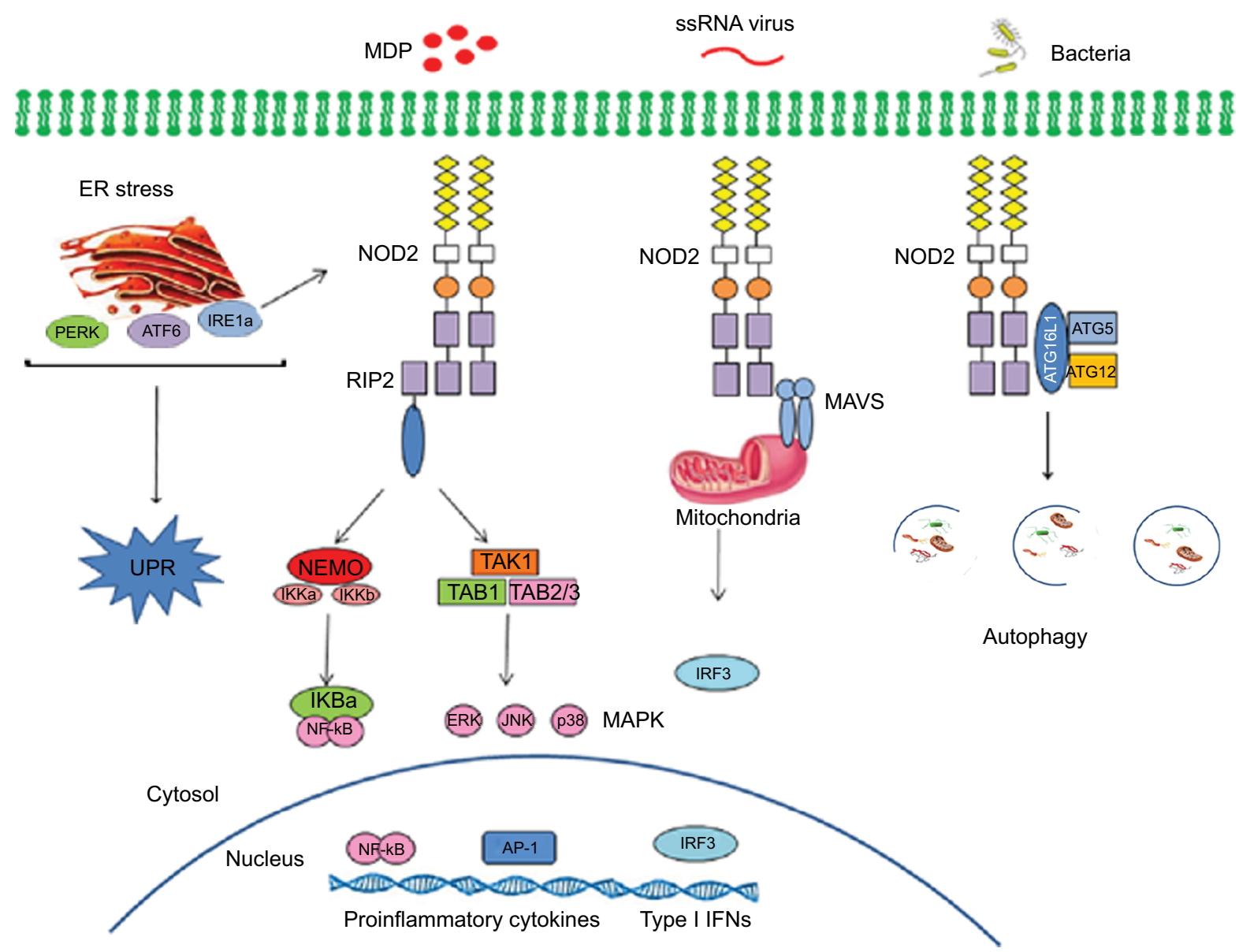

Figure 2 Signaling pathways triggered by NOD2.

Abbreviations: AP-I, activator protein-I; ATF6, activating transcription factor 6; ATG, autophagy-related genes; ATGI6LI, autophagy related I6 like I; ER, endoplasmic reticulum; ERK, extracellular signal-regulated kinase; IFNs, interferons; IKB, NF- $\mathrm{BB}$ inhibitor; IKK, IKB kinase; IRF3, interferon response factor 3; JNK, c-Jun N-terminal kinase; MAVS, mitochondrial antiviral signaling; MDP, muramyl dipeptide; NEMO, NF- KB essential modulator; NOD, nucleotide-binding oligomerization domain; PERK, protein kinase RNA-like endoplasmic reticulum kinase; RIP2, receptor-interacting protein kinase 2; TAB, TGF- $\beta$ activated kinase; TAKI, targets transforming growth factor$\beta$-activated kinase I; UPR, unfolded protein response. 
NOD2 pathway and regulators of intestinal inflammation. ${ }^{34,35}$ Interestingly, loss of Pellino 3 decreases RIP2 ubiquitination and activation of $\mathrm{NF}-\kappa \mathrm{B}$ and mitogen-activated protein kinases (MAPKs), while genetic X-linked inhibitor of apoptosis protein loss causes a blunted NOD2 response. ${ }^{34-36}$ Tripartite Motif Containing 27 negatively regulates NOD2 by ubiquitination, while the E3-ubiquitin ligase ZNRF4, degrading RIP2, is a negative regulator of NOD2-induced $\mathrm{NF}-\kappa \mathrm{B}$ activity as well. ${ }^{37,38}$ Differently, the ovarian tumor family deubiquitinase OTULIN was shown to dampen NOD2 signaling by increasing NF- $\kappa \mathrm{B}$ transcription. ${ }^{39}$

A recent study shows that leucine-rich repeat kinase 2 , whose polymorphisms have been associated with CD, leprosy and familiar Parkinson's disease, is a new RIP2-positive regulator by enhancing RIP2 phosphorylation upon NOD2 activation. $^{40}$

NOD2 signaling is known to be regulated by cytoskeleton elements also: the intermediate filament protein vimentin has been recently shown to interact with NOD2, in response to MDP, and affect NF- $\kappa \mathrm{B}$ induction. ${ }^{41}$

Large evidence supports the role of several microRNAs, including mir-320, miR-192, miR-122, miR-512, miR-671 and miR-495, as new important NOD2 regulators. ${ }^{42,43}$

\section{NOD2 genetics}

Hereditary polymorphisms in the gene encoding NOD2 have been associated with an increasing number of chronic inflammatory diseases, such as $\mathrm{CD}$, BS and EOS. ${ }^{6-8}$

In particular, the three main NOD2 polymorphisms, R702W (Arg702Trp), G908R (Gly908Arg) and L1007fsinsC, are highly associated with susceptibility to $C D .^{6,7,44} \mathrm{R} 702 \mathrm{~W}$ and G908R mutations are single amino acid changes within the LRR domain, whereas the L1007fsinsC mutation is caused by a deletion producing a reading frameshift that leads to the loss of 33 amino acids. ${ }^{6,7} \mathrm{CD}$-associated mutations result in NOD2 loss of functions, with a reduced responsiveness to MDP, enabling invasion of bacteria and abnormal mucosal immune response, which culminates in chronic intestinal inflammation. ${ }^{5-7}$

The variants in the NBD and in between the NBD and LRR are associated to BS, EOS and NOD2-associated autoinflammatory disease, respectively. , $^{8,45,46}$

At least 17 different mutations have been identified in the NBD domain of NOD2, ${ }^{45}$ of which the following four missense mutations are the most abundant: Arg334Glu (R334Q), Arg334Trp (R334W) and Leu469Phe (L469F), which together account for $80 \%$ of the cases, and Glu383Lys (E383K) (5\% of cases). ${ }^{47}$ Other NOD2 mutations, like the
Arg314Glu (R314Q) polymorphism that codes for a truncated form of the protein, have been described most rarely. ${ }^{8}$ These mutations are supposed to be gain of function, causing excessive NF- $\kappa \mathrm{B}$ and MAPK activation. ${ }^{9}$

\section{NOD2 functions}

\section{NOD2 and intestinal microbiota}

Humans are colonized by a collection of microbes, the largest numbers of which reside in the distal gut. The constant exposure of the intestinal tissue to gut microorganisms maintains the mucosa in a state of physiological inflammation, which balances tolerogenic and proinflammatory type responses to maintain homeostasis. ${ }^{48}$ Several studies highlight the essential role that NOD2 plays in maintaining the equilibrium between microbiome and host immune responses. ${ }^{49-51}$ An imbalance in this relationship results in dysbiosis, whereby pathogenic bacteria prevail on commensals, causing damage in the intestinal epithelial barrier as well as allowing bacterial invasion and inflammation. ${ }^{52,53}$ A negative feedback loop between NOD2 and commensal bacteria function has been described, whereby commensal bacteria promote NOD2 expression, which in turn prevents their over-expansion. ${ }^{52}$ Since NOD2 is an intracellular microbial sensor for gram-positive and gramnegative bacteria, it has been proposed that its deficiency or mutations can contribute to the modification of microbial composition and disease development in animal models. ${ }^{18,51,52}$

NOD2-deficient mice show a reduced number of intestinal intraepithelial lymphocytes impairing the epithelium integrity and leading to altered immune response to the resident microbiota. ${ }^{54}$ Moreover, NOD2- and RIP2-deficient mice show increased sensitivity to dextran sulfate sodium-induced colitis and colonic adenocarcinoma due to dysbiosis, which is transmitted to wild-type (WT) mice through the microbiota. ${ }^{55}$ NOD2 is also a critical regulator of microbiota in the small intestine. ${ }^{13}$ Indeed, NOD2 is highly expressed in Paneth cells, specialized cells located at the base of the intestinal crypts of Lieberkuhn, which are responsible for the ileal microbiota by secreting antimicrobial compounds, in response to bacterial products, including MDP, the agonist of NOD2..$^{13,18,56}$

There is evidence that NOD2 mutations increase the susceptibility to abnormal ileal inflammation. NOD2-deficient mice display Paneth and goblet cell dysfunction that promotes larger loads of bacteria in the ileum, including Bacteroides vulgatus, and excessive interferon- $\gamma$ (IFN $\gamma$ ) production. ${ }^{53,57}$

However, several studies do not support the evidence of a relationship between NOD2 mutations and altered microbiota composition, suggesting the contribution of other factors independent of the genotype, such as maternal microbiota 
transmission or environmental causes, but results are still controversial. ${ }^{58-60} \mathrm{NOD} 2$ participates in the recognition of a subset of pathogenic microorganisms that are able to invade and multiply intracellularly, such as Campylobacter, Citrobacter, Escherichia, Helicobacter, Listeria, Mycobacteria, Pseudomonas, Staphylococcus, Yersinia and other species. ${ }^{51,61,62}$ In a murine skin Staphylococcus aureus infection model, Nod2 $2^{-/-}$mice show skin lesions and increased bacterial numbers in the skin, compared to WT mice. ${ }^{63}$ Moreover, NOD2-deficient mice show a higher fecal bacterial load after Citrobacter rodentium infection, besides increased susceptibility to enteric Salmonella spp. and an impaired activation of Th17 cells after C. rodentium or Salmonella spp. exposure. ${ }^{64-66}$ Interestingly, NOD2L1007fsinsC mutants show a lower reactive oxygen species production and a reduced protection against bacterial invasion. ${ }^{67}$

NOD2 upregulation following vitamin D treatment results in increased killing of pneumococci in patients with frequent respiratory tract infections. ${ }^{68}$ Bacterial lipoproteintolerogenic macrophages show improved NOD1/NOD2dependent bactericidal activity to Sta. aureus and Salmonella typhimurium. ${ }^{69}$

NOD2 cooperates with other proteins for defense against pathogens, such as the autophagic protein ATG16L1 and the oxidase dual oxidase 2 that represents a major source of NOD2-dependent bactericidal reactive oxygen species generation. $^{70}$

\section{NOD2 in innate and adaptive immunity}

NOD2 drives the innate inflammatory response to bacteria and viruses through the activation of NF- $\mathrm{B}$, MAPK and caspase-1 pathways, which results in increased expression of proinflammatory factors, including IL-1 $\beta$, tumor necrosis factor-alpha (TNF $\alpha$ ), IL-6, IL-12p40, CC-chemokine ligand 2, the neutrophil chemoattractants $\mathrm{CXC}$-chemokine ligand 8 (also known as IL-8), CXC-chemokine ligand 2 as well as various antimicrobial agents, as defensins, and in recruitment and priming of neutrophils, inflammatory monocytes and dendritic cells. ${ }^{64,71-73}$

NOD2 also activates other signaling pathways. Upon binding the bacterial ligand, NOD2 induces recruitment of RIP2, which binds TNF receptor-associated factor (TRAF) 3 that activates the TANK binding kinase 1 and IKKe, which phosphorylates and activates regulatory interferon response factor 3 (IRF3). Activated IRF3 passes into the nucleus, where it binds with the molecules of IFN-stimulated response elements inducing the IFN gene expression of type $\mathrm{I}^{74,75}$
Moreover, NOD2 triggers Notch1 signaling response. Crosstalk between NOD2 and its novel downstream effector Notch1-PI3K in the macrophages contributes to macrophage survival, reducing TNF $\alpha / \mathrm{IFN} \gamma$-induced apoptosis, and modulates the expression of IL-10 and a battery of genes associated with anti-inflammatory functions. ${ }^{76}$

In addition to its main role in innate immunity, NOD2 is capable of activating the adaptive immune system; indeed, it is a key driver of T helper (Th) 2-type immunity resulting in IL-4 and IL-5 production. ${ }^{77}$ Full Th2 induction upon Nod2 activation is dependent on both thymic stromal lymphopoietin production by the stromal cells and the upregulation of the costimulatory molecule, OX40 ligand, on the dendritic cells. ${ }^{77}$ Several studies have shown that co-stimulation with NOD2 and toll-like receptor (TLR) agonists induces a synergistic production of Th1-associated cytokines in different types of cells, although the mechanism is still unclear. ${ }^{78,79}$

Finally, MDP-induced NOD2 activation has been shown to promote the development of Th17 cells and the consequent production of IL-17A, IL-17F, IL-21 and IL-22. ${ }^{80,81}$

\section{NOD2 and antiviral response}

The innate immune system detects viral infections through the recognition of virus-associated PAMPs, such as genomic DNA, RNA or dsRNA produced in infected cells, which are recognized by the PRRs expressed in innate immune cells. ${ }^{82}$ After recognition of the viral components, PRRs activate an antiviral response that results in the production of type I IFNs, proinflammatory cytokines, eicosanoids and chemokines and subsequent induction of adaptive immune response. ${ }^{82}$ Current literature evidences that NOD2 is also able to control virus infections. ${ }^{83,84}$ Indeed, the antiviral SB 9200, a novel first-in-class oral modulator of innate immunity with a broadspectrum activity in vitro and in vivo against RNA viruses including hepatitis $\mathrm{C}$ virus, norovirus, respiratory syncytial virus and hepatitis B virus, is also believed to act via NOD2 pathway. ${ }^{85}$ Furthermore, infection with human cytomegalovirus results in significant induction of NOD2 expression, activating downstream NF- $\kappa \mathrm{B}$ and IFN pathways. ${ }^{84}$ Besides, following recognition of a viral ssRNA genome, NOD2 uses the adaptor mitochondrial antiviral signaling protein to activate IRF3; interestingly, NOD2-deficient mice fail to produce IFN efficiently. Similar roles for NOD2 are observed in response to influenza $A$ and parainfluenza viruses. ${ }^{75}$

NOD2-induced triggering of NF- $\kappa B$ and IRF3 in response to viral infection is also interesting since it has been shown that a coordinated activation of NF- $\kappa \mathrm{B}$ and IRF3 pathways synergistically promotes optimal IFN expression, including 
IFN- $\beta$, and hence the antiviral resistance. ${ }^{86}$ Moreover, a regulatory role of NOD2 in enhancing the function of RNase-L through the binding with $2^{\prime}-5^{\prime}$-oligoadenylate synthetase type 2, a dsRNA binding protein, has been described as an additional strategy to induce an innate immune response to control bacteria and viruses. ${ }^{87}$

\section{NOD2 and autophagy}

Autophagy represents a cellular stress response that plays key roles in physiological processes, such as innate and adaptive immunity, adaptation to starvation, degradation of misfolded or aggregated proteins or damaged organelles and elimination of intracellular pathogens, in order to promote cellular survival. The role of NOD1 and NOD2 in autophagy is a recent discovery and still under debate.

Travassos et al suggested that, by a mechanism independent of the adaptor RIP2 and transcription factor NF- $\mathrm{KB}, \mathrm{NOD} 1$ and NOD2 recruited the autophagy protein ATG16L1, an essential component of the autophagic machinery, to the plasma membrane at the bacterial entry site. ${ }^{25}$ Moreover, in cells homozygous for the CD-associated NOD2 frameshift mutation, mutant NOD2 failed to recruit ATG16L1 to the membrane and wrapping of invading bacteria by autophagosomes was impaired. ${ }^{25}$ Furthermore, knockdown of ATG16L1 resulted in specific upregulation of NOD2 response, establishing a role of ATG16L1 as a negative regulator of the NOD2/RIP2 pathway. ${ }^{88}$ NOD2-mediated autophagy is crucial to hold intramucosal bacterial burden and limit intestinal inflammation. ${ }^{89-91}$

Interestingly, ATG16L1 and NOD2 single nucleotide polymorphisms are both implicated in increased susceptibility to $\mathrm{CD} .^{91-94}$

More recently, other authors have provided evidence of an alternative mechanism of NOD2-dependent autophagy activation which requires RIP2. Cooney et al have shown that NOD2 triggering by MDP induces autophagy in dendritic cells and this effect requires RIP $2 .{ }^{95}$ Anand et al demonstrated that macrophages deficient in the TLR2 and NOD/RIP2 pathway display defective autophagy induction in response to Listeria monocytogenes. ${ }^{96}$ Homer et al have shown a dual role for RIP2 tyrosine kinase activity in NOD2-dependent autophagy: RIP2 both sends a positive autophagy signal through activation of $\mathrm{p} 38 \mathrm{MAPK}$ and relieves repression of autophagy mediated by the phosphatase PP2A. ${ }^{97}$

\section{NOD2 and endoplasmic reticulum (ER) stress}

The ER stress, activated by accumulation of unfolded or misfolded proteins and microbial infections, triggers a host response known as the unfolded protein response (UPR), which involves the activation of three transmembrane receptors: activating transcription factor 6 , protein kinase RNAlike ER kinase and inositol-requiring enzyme $1 \alpha$ (IRE $1 \alpha)$. Once activated, IRE1 $\alpha$ recruits TRAF2 to the ER membrane to initiate inflammatory responses via c-Jun N-terminal kinase (JNK) pathway and NF- $\mathrm{KB} .{ }^{98} \mathrm{NOD} 2$ was found to transduce ER stress signals to elicit inflammation. ${ }^{99,100}$ Indeed, TRAF2 has been shown to interact with NOD1 and NOD2 to orchestrate this inflammatory branch of UPR, which also requires the adaptor protein RIP2. ${ }^{9}$ However, the mechanism by which ER stress, and more specifically IRE1 $\alpha$, activates NOD1/NOD2 signaling is still unclear. Recent work provides evidence that the ER stress inducers thapsigargin and dithiothreitol trigger production of the proinflammatory cytokine IL-6 in a NOD1/2-dependent fashion. ${ }^{100,101}$ In vivo experiments confirmed the in vitro observations by showing that systemic proinflammatory responses induced by thapsigargin administration were blunted in $N O D 1^{-/-} N O D 2^{-/-}$mice. ${ }^{100}$

Several UPR-related genes have been identified as inflammatory bowel disease (IBD) risk loci. ${ }^{102,103}$ In particular, new evidence has linked the UPR and autophagy in Paneth cells to the development of CD-like transmural ileitis. ${ }^{104}$ The genetic convergence of genetic polymorphisms on innate immune pathways, such as NOD2, autophagy and ER stress, may open novel therapeutic options for the treatment of intestinal inflammation. ${ }^{105,106}$ The involvement of ER stress and NOD2 in chronic inflammatory diseases, including IBD and type 2 diabetes, has important implications for understanding the pathogenesis and for the management of these diseases. ${ }^{107,108}$

\section{NOD2 and IBD}

IBD is a group of chronic multifactorial disorders that includes $\mathrm{CD}$, characterized by transmural inflammation that can affect any region of the gastrointestinal tract and ulcerative colitis that results in inflammation and ulcers of the colon and rectum. The etiology of IBD has yet to be fully elucidated; however, it is postulated that it is the result of an unbalanced crosstalk between gut luminal content and the mucosal immune system in genetically susceptible hosts. ${ }^{109}$

Recent genome-wide association studies have revealed 163 susceptibility loci for IBD. ${ }^{110}$ NOD2 was the first gene identified as a risk factor for ileal CD. ${ }^{6,7}$

Three NOD2 polymorphisms in the LRR region are directly associated with $\mathrm{CD}$, of which the most known is the frameshift mutation (L1007fs), whereas the other two are missense mutations (R702W and G908R) ${ }^{6,7}$ It is postulated that the LRR domain of CD-associated variants is likely 
to be impaired, possibly to various degrees, in recognizing microbial components and/or in physiologically inhibiting NOD2 dimerization, thus resulting in the inappropriate activation of NF- $\mathrm{KB}$ in monocytes, bacterial clearance and impairment of intestinal permeability. ${ }^{6,111,112}$ Thus, individuals who are heterozygous for NOD2 variants have a 2-4-fold increased risk of developing $\mathrm{CD}$, whereas homozygous variants have an additional risk of 20-40-fold. ${ }^{6,7113}$ However, many NOD2 variant carriers do not develop the disease, suggesting that other factors are involved in disease onset. ${ }^{11,112}$ Similarly, NOD2-knockout mice lack symptoms associated with spontaneous intestinal inflammation. ${ }^{114}$ Nevertheless, NOD2-deficient mice demonstrate defects in mediating antibacterial responses, such as increased systemic Listeria colonization, suggesting decreased antimicrobial peptides in their intestine. ${ }^{18}$ Moreover, NOD2-deficient mice show altered gut permeability. ${ }^{49}$ This evidence is also supported by Amendola et al who attributed the absence of spontaneous colonic inflammation in NOD2-deficient mice to permeability changes: the latter may increase the exposure of dendritic cells to factors, such as TLR ligands, that influence T-reg cell development and subsequent changes in the microbiota. ${ }^{115}$

Homozygous/compound heterozygous carriage of the CD-associated NOD2 variants has been reported to be significantly associated with ileal disease involvement, stricturing/ stenosing disease behavior and 2-3 years earlier age of disease onset compared to NOD2 WT CD patients. ${ }^{16,117}$ Ileal CD patients with NOD2 L1007fs mutation showed a reduced release of $\alpha$-defensin from Paneth cells. ${ }^{118,119}$

Interestingly, the reduction of $\alpha$-defensin release seems to be a secondary effect of the NOD2 knockdown, causing excessive inflammation and loss of Paneth cell. However, the question remains controversial. ${ }^{120}$

Notably, CD-associated NOD2 variants are defective in ATG16L1 recruitment and exhibit an altered autophagy in a cell type-specific manner. ${ }^{91}$ Furthermore, in patients with ileal $\mathrm{CD}$, mutations in autophagy genes other than in NOD2 lead to an impaired secretion of Paneth cell-derived $\alpha$-defensins with a deficiency in clearance of internalized bacteria. ${ }^{119-122}$ Therefore, the combination of defective innate immune responses by NOD2 and ineffective bacterial clearance by autophagy could together be responsible for $\mathrm{CD}$ development and progression.

\section{NOD2 and extraintestinal diseases}

A consistent issue in human genetics is that genes implicated in one disorder could potentially increase susceptibility in other related disorders. It has been shown that three major missense mutations in the NBD domain of NOD2, R334Q, R334W and L469F are involved in an extremely rare, monogenic dominant disorder characterized by granulomatous inflammatory arthritis, uveitis and dermatitis, known as BS. ${ }^{45,123,124}$ The latter is related to gain-of-function mutations as opposed to $\mathrm{CD}$ mutations which appear to be recessive and are characterized with respect to NF- $\kappa \mathrm{B}$ activation by a loss of function. ${ }^{44}$ More rarely, the NOD2 R314Q polymorphism, coding for a truncated form of the protein, has also been found to be associated with $\mathrm{BS}^{8}$

NOD2 mutations, leading to increased activity of NF- $\kappa B$, have been associated to EOS, a multisystemic granulomatous disease characterized by arthritis, uveitis and cutaneous involvement. ${ }^{125}$ NOD2 mutations and similar clinical and histologic characteristics suggest a link between EOS and BS., ${ }^{9,47}$

Increased NOD2 expression has been found in patients with rheumatoid arthritis, a chronic inflammatory disorder that affects the joints, causing structural deformities. ${ }^{126}$ Upon stimulation with MDP, peripheral blood mononuclear cells isolated from rheumatoid arthritis patients produced high amounts of TNF $\alpha$, IL-8 and IL-1 $\beta$, while NOD2 downregulation significantly decreased proinflammatory cytokines, NF- $\kappa B$, TRAF6 and IKK levels. ${ }^{127}$

Clinical and experimental studies have recently shown a role of NOD2 in cardiovascular diseases inducing vascular inflammation and severity of atherosclerosis, the most common pathologic process of coronary artery and cerebrovascular disease. ${ }^{128}$ NOD2 has been localized in inflamed areas of atherosclerotic lesions and is overexpressed in endothelial cells delimiting the lumen of diseased vessels. ${ }^{129}$ Moreover, NOD2-mediated IL-6, IL- 8 and IL-1 $\beta$ production induces vascular inflammation and promotes expansion of the lipidrich necrotic areas. ${ }^{130}$

NOD2 mRNA has also been found to be highly expressed in bronchoalveolar lavage and in peripheral blood mononuclear cells from patients with Behcet's disease, a rare disorder with unknown etiology, characterized by a systemic vasculitis and pulmonary manifestations. ${ }^{131,132} \mathrm{~A}$ recent study has shown that NOD2 aggravates myocardial ischemia/ reperfusion injury by inducing cardiomyocyte apoptosis and inflammation through JNK, p38MAPK and NF- $\mathrm{KB}$ signaling in mice and has suggested NOD2 as a potential target for the treatment of the disease. ${ }^{133}$ Furthermore, NOD2 has been reported to be involved in IL-6, IL- 8 and monocyte chemoattractant protein-1 production induced by the invasion of human aortic endothelial cells by Streptococcus mutans, the primary etiologic agent of dental caries, associated with the development of cardiovascular disease. ${ }^{134}$ 
NOD2 variants have been associated with the systemic autoinflammatory disease (NOD2-associated autoinflammatory disease), a genetically complex multisystem disorder characterized by periodic fever, dermatitis, arthritis and gastrointestinal and sicca-like symptoms, but with a phenotypic and genotypic profile distinct from $\mathrm{CD} .{ }^{135}$

NOD2 polymorphisms have been also related to atopic dermatitis, a chronically relapsing inflammatory skin disease associated with basophil infiltration and an exacerbation of inflammation by Sta. aureus. ${ }^{136-138}$

Recent literature describes a central role of NOD2 in susceptibility to obesity and metabolic dysfunction. ${ }^{139}$ NOD2-deficient mice show increased bacterial adherence to the intestinal mucosa and bacterial infiltration in metabolic tissues, such as hepatic and adipose tissue, exacerbating inflammation and insulin resistance. ${ }^{140}$ Moreover, NOD2-/BALB/c mice have shown susceptibility to obesity, hyperlipidemia, hyperglycemia, glucose intolerance, increased adiposity and hepatic steatosis, as compared to WT mice. ${ }^{141}$

Finally, NOD2-increased expression has been observed in patients with active Vogt-Koyanagi-Harada disease, a rare granulomatous inflammatory disease that affects pigmented structures, promoting proinflammatory cytokine production and Th1 and Th17 cells stimulation. ${ }^{142}$

\section{NOD2 and cancer}

NOD2 has been associated to cancer development. Data from animal models indicate that NOD2 deficiency leads to dysbiosis resulting in increased risk of colitis and colitis associated colorectal cancer. ${ }^{143} \mathrm{~A}$ recent study in NOD2- or RIP2-deficient mice shows an increased epithelial dysplasia following dextran sulfate sodium-induced colitis, suggesting a NOD2-protective role in cancer development via regulation of gut bacterial equilibrium. ${ }^{55}$

Emerging evidence in human studies implies that several NOD2 polymorphisms may influence individual susceptibility to cancer. ${ }^{143,144}$ However, the results are still inconclusive due to confounding genetic, bacterial and environmental factors that may alter variant allele penetrance or the differences in sample size, geographic variation and genotyping methods. In general, NOD2 gene polymorphisms are associated with altered risk of gastric, colorectal, breast, ovarian, prostate, testicular, lung, laryngeal, liver, gallbladder, biliary tract, pancreatic, small bowel, kidney, urinary bladder cancer, skin cancer, non-thyroid endocrine tumors, lymphoma and leukemia. ${ }^{145}$ In particular, the three most common NOD2 polymorphisms, rs2066844 C/T (R702W), rs2066845 C/G (G908R) and rs2066847 (3020insC), have been associated with increased risk of gastrointestinal cancer. ${ }^{146}$ Furthermore, NOD2 has also been involved in the development of gastric cancer induced by Helicobacter pylori. ${ }^{144}$

\section{Conclusion}

The involvement of NOD2 in the pathogenesis of several genetic diseases indicates that this protein is a key regulator of immune and inflammatory responses. Extensive studies have evidenced a fundamental role of NOD2 in maintaining the equilibrium between bacteria, epithelia and innate immune response of the host. This protective function is lost in case of NOD2 mutations, resulting in exacerbated inflammation and the onset of various diseases. On the other hand, inappropriate activation of WT NOD2 is reported in many chronic inflammatory diseases, resulting in continuous production of proinflammatory mediators, but the exact mechanism underlying this NOD2 dysregulation is not yet well established. Recent literature has shifted the interest from genetic to epigenetic control and to interactions with other innate immune pathways such as autophagy and ER stress. Many questions remain unanswered, including the relation between NOD2 mutations and microbiota and the understanding of the processes by which mutations in the NOD2 could be associated with the susceptibility to inflammation and development of diseases. When the exact mechanism of regulation and functions of NOD2 will be unraveled, it could lead to the development of more effective therapies for inflammatory disorders.

\section{Disclosure}

The authors report no conflicts of interest in this work.

\section{References}

1. Takeuchi $\mathrm{O}$, Akira S. Pattern recognition receptors and inflammation. Cell. 2010;140(6):805-20.

2. Proell M, Riedl SJ, Fritz JH, Rojas AM, Schwarzenbacher R. The Nod-like receptor (NLR) family: a tale of similarities and differences. PLoS One. 2008;3(4):e2119.

3. Kawai T, Akira S. The roles of TLRs, RLRs and NLRs in pathogen recognition. Int Immunol. 2009;21(4):317-337.

4. Feerick CL, McKernan DP. Understanding the regulation of pattern recognition receptors in inflammatory diseases - a 'Nod' in the right direction. Immunology. 2017;150(3):237-247.

5. Philpott DJ, Sorbara MT, Robertson SJ, Croitoru K, Girardin SE. NOD proteins: regulators of inflammation in health and disease. Nat Rev Immunol. 2014;14(1):9-23.

6. Hugot JP, Chamaillard M, Zouali H, et al. Association of NOD2 leucine rich repeat variants with susceptibility to Crohn's disease. Nature. 2001;411(6837):599-603.

7. Ogura Y, Bonen DK, Inohara N, et al. A frameshift mutation in NOD2 associated with susceptibility to Crohn's disease. Nature. 2001;411(6837):603-606.

8. Dugan J, Griffiths E, Snow P et al. Blau syndrome-associated Nod2 mutation alters expression of full length NOD2 and limits responses to muramyl dipeptide in knock-in mice. J Immunol. 2015;194(1):349-357. 
9. Kanazawa N, Okafuji I, Kambe N, et al. Early-onset sarcoidosis and CARD15 mutations with constitutive nuclear factor-kappaB activation: common genetic etiology with Blau syndrome. Blood. 2005;105(3): 1195-1197.

10. Tattoli I, Carneiro LA, Jéhanno M, et al. NLRX1 is a mitochondrial NOD-like receptor that amplifies NF-kappaB and JNK pathways by inducing reactive oxygen species production. EMBO Rep. 2008;9(3):293-300.

11. Moore CB, Bergstralh DT, Duncan JA et al. NLRX1 is a regulator of mitochondrial antiviral immunity. Nature. 2008;451(7178):573-577.

12. Boyle JP, Parkhouse R, Monie TP. Insights into the molecular basis of the NOD2 signalling pathway. Open Biol. 2014;4(12):140178.

13. Sidiq T, Yoshihama S, Downs I, Kobayashi KS. Nod2: a critical regulator of ileal microbiota and Crohn's disease. Front Immunol. 2016;7:367. eCollection 2016.

14. Nigro G, Rossi R, Commere PH, Jay P, Sansonetti PJ. The cytosolic bacterial peptidoglycan sensor Nod2 affords stem cell protection and links microbes to gut epithelial regeneration. Cell Host Microbe. 2014;15(6):792-798.

15. Philpott DJ, Girardin SE. Nod-like receptors: sentinels at host membranes. Curr Opin Immunol. 2010;22(4):428-434.

16. Hofmann K, Bucher P, Tschopp J. The CARD domain: a new apoptotic signaling motif. Trends Biochem Sci. 1997;22(5):155-166.

17. Girardin SE, Boneca IG, Viala J, et al. Nod2 is a general sensor of peptidoglycan through muramyl dipeptide (MDP) detection. J Biol Chem. 2003;278(11):8869-8872.

18. Kobayashi KS, Chamaillard M, Ogura Y, et al. Nod2-dependent regulation of innate and adaptive immunity in the intestinal tract. Science. 2005;307(5710):731-734.

19. Park JH, Kim YG, McDonald C, et al. RICK/RIP2 mediates innate immune responses induced through Nod1 and Nod2 but not TLRs. J Immunol. 2007;178(4):2380-2386.

20. Rahighi S, Ikeda F, Kawasaki M, et al. Specific recognition of linear ubiquitin chains by NEMO is important for NF-kappaB activation Cell. 2009;136(6):1098-1109.

21. Jiang $X$, Chen ZJ. The role of ubiquitylation in immune defence and pathogen evasion. Nat Rev Immunol. 2011;12(1):35-48.

22. Karin M. The Regulation of AP-1 Activity by Mitogen-activated Protein Kinases. J Biol Chem. 1995;270(28):16483-16486.

23. Hsu LC, Ali SR, McGillivray S, et al. A NOD2-NALP1 complex mediates caspase-1-dependent IL-1beta secretion in response to Bacillus anthracis infection and muramyl dipeptide. Proc Natl Acad Sci USA. 2008;105(22):7803-7808.

24. Meinzer U, Barreau F, Esmiol-Welterlin S, et al. Yersinia pseudotuberculosis effector YopJ subverts the Nod2/RICK/TAK1 pathway and activates caspase-1 to induce intestinal barrier dysfunction. Cell Host Microbe. 2012;11(4):337-351.

25. Travassos LH, Carneiro LA, Ramjeet M, et al. Nod1 and Nod2 direct autophagy by recruiting ATG16L1 to the plasma membrane at the site of bacterial entry. Nat. Immunol. 2010;11(1):55-62.

26. Warner N, Burberry A, Franchi L, et al. A genome-wide siRNA screen reveals positive and negative regulators of the NOD2 and NF- $\kappa B$ signaling pathways. Sci Signal. 2013;6(258):rs3.

27. Richmond AL, Kabi A, Homer CR, et al. The nucleotide synthesis enzyme CAD inhibits NOD2 antibacterial function in human intestinal epithelial cells. Gastroenterology. 2012;142(7):1483-1492.

28. Lecat A, Di Valentin E, Somja J, et al. The c-Jun N-terminal kinase (JNK)-binding protein (JNKBP1) acts as a negative regulator of NOD2 protein signaling by inhibiting its oligomerization process. J Biol Chem. 2012;287(35):29213-29226.

29. Lipinski S, Grabe N, Jacobs G, et al. RNAi screening identifies mediators of NOD2 signaling: implications for spatial specificity of MDP recognition. Proc Natl Acad Sci USA. 2012;109(52):21426-21431.

30. Lee KH, Biswas A, Liu YJ, Kobayashi KS. Proteasomal degradation of Nod2 protein mediates tolerance to bacterial cell wall components. J Biol Chem. 2012;287(47):39800-39811.
31. Watanabe T, Asano N, Meng G, et al. NOD2 downregulates colonic inflammation by IRF4-mediated inhibition of K63-linked polyubiquitination of RICK and TRAF6. Mucosal Immunol. 2014;7(6):1312-1325.

32. Mohanan V, Grimes CL. The molecular chaperone HSP70 binds to and stabilizes NOD2, an important protein involved in Crohn disease. J Biol Chem. 2014;289(27):18987-18998.

33. Tigno-Aranjuez JT, Abbott DW. Ubiquitination and phosphorylation in the regulation of NOD2 signaling and NOD2-mediated disease. Biochim Biophys Acta. 2012;1823(11):2022-2028.

34. Yang S, Wang B, Humphries F, et al. Pellino3 ubiquitinates RIP2 and mediates Nod2-induced signaling and protective effects in colitis. Nat Immunol. 2013;14(9):927-936.

35. Damgaard RB, Nachbur U, Yabal M, et al. The ubiquitin ligase XIAP recruits LUBAC for NOD2 signaling in inflammation and innate immunity. Mol Cell. 2012;46(6):746-758.

36. Chirieleison SM, Marsh RA, Kumar P, Rathkey JK, Dubyak GR, Abbott DW. Nucleotide-binding oligomerization domain (NOD) signaling defects and cell death susceptibility cannot be uncoupled in $\mathrm{X}$-linked inhibitor of apoptosis (XIAP)-driven inflammatory disease. J Biol Chem. 2017;292(23):9666-9679.

37. Zurek B, Schoultz I, Neerincx A, et al. TRIM27 negatively regulates NOD2 by ubiquitination and proteasomal degradation. PLoS One. 2012;7(7):e41255.

38. Bist P, Cheong WS, Ng A, et al. E3 Ubiquitin ligase ZNRF4 negatively regulates NOD2 signalling and induces tolerance to MDP. Nat Commun. 2017;8:15865.

39. Fiil BK, Damgaard RB, Wagner SA, et al. OTULIN restricts Met1linked ubiquitination to control innate immune signaling. Mol Cell. 2013;50(6):818-830

40. Yan R, Liu Z. LRRK2 enhances Nod1/2-mediated inflammatory cytokine production by promoting Rip2 phosphorylation. Protein Cell. 2017;8(1):55-66.

41. Stevens $C$, Henderson $P$, Nimmo ER, et al. The intermediate filament protein, vimentin, is a regulator of NOD2 activity. Gut. 2013;62(5), 695-707.

42. Pierdomenico M, Cesi V, Cucchiara S, et al. NOD2 is regulated by Mir320 in physiological conditions but this control is altered in inflamed tissues of patients with inflammatory bowel disease. Inflamm Bowel Dis. 2016;22(2):315-326.

43. Chuang AY, Chuang JC, Zhai Z, Wu F, Kwon JH. NOD2 expression is regulated by microRNAs in colonic epithelial HCT116 cells. Inflamm Bowel Dis. 2014;20(1):126-35.

44. Strober W, Asano N, Fuss I, Kitani A, Watanabe T. Cellular and molecular mechanisms underlying NOD2 risk-associated polymorphisms in Crohn's disease. Immunol Rev. 2014;260(1):249-260.

45. Miceli-Richard C, Lesage S, Rybojad M, et al. CARD15 mutations in Blau syndrome. Nat Genet. 2001;29(1):19-20.

46. Yao Q. Nucleotide-binding oligomerization domain containing 2: structure, function, and diseases. Semin Arthritis Rheum. 2013;43(1):125-130.

47. Caso F, Galozzi P, Costa L, Sfriso P, Cantarini L, Punzi L. Autoinflammatory granulomatous diseases: from Blau syndrome and early-onset sarcoidosis to NOD2-mediated disease and Crohn's disease. RMD Open. 2015;1(1):e000097.

48. Blander JM, Longman RS, Iliev ID, Sonnenberg GF, Artis D. Regulation of inflammation by microbiota interactions with the host. Nat Immunol. 2017;18(8):851-860.

49. Balasubramanian I, Gao N. From sensing to shaping microbiota: insights into the role of NOD2 in intestinal homeostasis and progression of Crohn's disease. Am J Physiol Gastrointest Liver Physiol. 2017;313(1): G7-G13.

50. Rehman A, Sina C, Gavrilova O, et al. Nod2 is essential for temporal development of intestinal microbial communities. Gut. 2011;60(10): 1354-1362.

51. Al Nabhani Z, Dietrich G, Hugot JP, Barreau F. Nod2: the intestinal gate keeper. PLoS Pathog. 2017;13(3):e1006177. 
52. Biswas A, Petnicki-Ocwieja T, Kobayashi KS. Nod2: a key regulator linking microbiota to intestinal mucosal immunity. J Mol Med (Berl). 2012;90(1):15-24

53. Ramanan D, Tang MS, Bowcutt R, Loke P, Cadwell K. Bacterial sensor Nod2 prevents inflammation of the small intestine by restricting the expansion of the commensal Bacteroides vulgatus. Immunity. 2014;41(2):311-324.

54. Jiang W, Wang X, Zeng B, et al. Recognition of gut microbiota by NOD2 is essential for the homeostasis of intestinal intraepithelial lymphocytes. J Exp Med. 2013;210(11):2465-2476.

55. Couturier-Maillard A, Secher T, Rehman A, et al. NOD2-mediated dysbiosis predisposes mice to transmissible colitis and colorectal cancer. J Clin Invest. 2013;123(2):700-711.

56. Ogura Y, Lala S, Xin W, et al. Expression of NOD2 in Paneth cells: a possible link to Crohn's ileitis. Gut. 2003;52(11):1591-1597.

57. Petnicki-Ocwieja T, Hrncir T, Liu YJ, et al. Nod2 is required for the regulation of commensal microbiota in the intestine. Proc Natl Acad Sci USA. 2009;106(37):15813-15818.

58. Robertson SJ, Zhou JY, Geddes K, et al. Nod1 and Nod2 signaling does not alter the composition of intestinal bacterial communities at homeostasis. Gut Microbes. 2013;4(3):222-231.

59. Robertson SJ, Geddes K, Maisonneuve C, Streutker CJ, Philpott DJ. Resilience of the intestinal microbiota following pathogenic bacterial infection is independent of innate immunity mediated by NOD1 or NOD2. Microbes Infect. 2016;18(7-8):460-471.

60. Shanahan MT, Carroll IM, Grossniklaus E, et al. Mouse Paneth cell antimicrobial function is independent of Nod2. Gut. 2014;63(6): 903-910.

61. Bereswill S, Grundmann U, Alutis ME, Fischer A, Heimesaat MM. Campylobacter jejuni infection of conventionally colonized mice lacking nucleotide-oligomerization-domain-2. Gut Pathog. 2017;9:5.

62. Caruso R, Warner N, Inohara N, Núñez G. NOD1 and NOD2: signaling, host defense, and inflammatory disease. Immunity. 2014;41(6):898-908.

63. Hruz P, Zinkernagelb AS, Jenikovaa G, et al. NOD2 contributes to cutaneous defense against Staphylococcus aureus through $\alpha$-toxindependent innate immune activation. Proc Natl Acad Sci USA. 2009;106(31):12873-12878.

64. Kim YG, Kamada N, Shaw MH, et al. The NOD2 sensor promotes intestinal pathogen eradication via the chemokine CCL2dependent recruitment of inflammatory monocytes. Immunity. 2011;34(5):769-780.

65. Geddes K, Rubino S, Streutker C, et al. NOD1 and NOD2 regulation of inflammation in the salmonella colitis model. Infect. Immun. 2010;78(12):5107-5115.

66. Geddes K, Rubino SJ, Magalhaes JG, et al. Identification of an innate T helper type 17 response to intestinal bacterial pathogens. Nat Med. 2011;17(7):837-844.

67. Lecat,A, Piette J, Legrand-Poels S. The protein Nod2: an innate receptor more complex than previously assumed. Biochem Pharmacol. 2010;80(12):2021-2031.

68. Subramanian K, Bergman P, Henriques-Normark B. Vitamin D promotes pneumococcal killing and modulates inflammatory responses in primary human neutrophils. J Innate Immun. 2017;9(4):375-386.

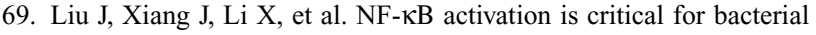
lipoprotein tolerance-enhanced bactericidal activity in macrophages during microbial infection. Sci Rep. 2017;7:40418.

70. Lipinski S, Till A, Sina C, et al. DUOX2-derived reactive oxygen species are effectors of NOD2-mediated antibacterial responses. Cell Sci. 2009;122(Pt 19):3522-3530.

71. Beynon V, Cotofana S, Brand S, et al. NOD2/CARD15 genotype influences MDP-induced cytokine release and basal IL-12p40 levels in primary isolated peripheral blood monocytes. Inflamm Bowel Dis. 2008;14(8):1033-1040.

72. Fritz JH, Girardin SE, Fitting C, et al. Synergistic stimulation of human monocytes and dendritic cells by Toll-like receptor 4 and NOD1- and NOD2-activating agonists. Eur J Immunol. 2005;35(8):2459-2470.
73. Coulombe F, Fiola S, Akira S, Cormier Y, Gosselin J. Muramyl dipeptide induces NOD2-dependent Ly6 $\mathrm{C}^{\text {high }}$ monocyte recruitment to the lungs and protects against influenza virus infection. PLoS One. 2012;7(5):e36734.

74. Kapoor A, Fan YH, Arav-Boger R. Bacterial muramyl dipeptide (MDP) restricts human cytomegalovirus replication via an IFN- $\beta$-dependent pathway. Sci Rep. 2016;6:20295.

75. Sabbah A, Chang TH, Harnack R, et al. Activation of innate immune antiviral responses by Nod2. Nat. Immunol. 2009;10(10):1073-1080.

76. Bansal K, Balaji KN. Intracellular pathogen sensor NOD2 programs macrophages to trigger Notch1 activation. J Biol Chem. 2011;286(7):5823-5835.

77. Magalhaes JG, Rubino SJ, Travassos LH, et al. Nucleotide oligomerization domain-containing proteins instruct $\mathrm{T}$ cell helper type 2 immunity through stromal activation. Proc Natl Acad Sci U S A. 2011;108(36):14896-14901.

78. Tada H, Aiba S, Shibata K, Ohteki T, Takada H. Synergistic effect of Nod1 and Nod2 agonists with toll-like receptor agonists on human dendritic cells to generate interleukin-12 and T helper type 1 cells. Infect Immun. 2005;73(12):7967-7976.

79. Jeong YJ, Kang MJ, Lee SJ, et al. Nod2 and Rip2 contribute to innate immune responses in mouse neutrophils. Immunology. 2014;143(2):269-276.

80. Brain O, Owens BM, Pichulik T, et al. The intracellular sensor NOD2 induces microRNA-29 expression in human dendritic cells to limit IL-23 release. Immunity. 2013;39(3):521-536.

81. Van Beelen AJ, Zelinkova Z, Taanman-Kueter EW, et al. Stimulation of the intracellular bacterial sensor NOD2 programs dendritic cells to promote interleukin-17 production in human memory $\mathrm{T}$ Cells. Immunity. 2007;27(4):660-669.

82. Kawai T, Akira S. Innate immune recognition of viral infection. Nat Immunol. 2006;7(2):131-137.

83. Keestra-Gounder AM, Tsolis RM. NOD1 and NOD2: beyond peptidoglycan sensing. Trends Immunol. 2017;38(10):758-767.

84. Kapoor A, Forman M, Arav-Boger R. Activation of nucleotide oligomerization domain 2 (NOD2) by human cytomegalovirus initiates innate immune responses and restricts virus replication. PLoS One. 2014;9(3):e92704.

85. Jones M, Cunningham ME, Wing P, et al. SB 9200, a novel agonist of innate immunity, shows potent antiviral activity against resistant HCV variants. J Med Virol. 2017;89(9):1620-1628.

86. Wathelet MG, Lin CH, Parekh BS, Ronco LV, Howley PM, Maniatis T. Virus infection induces the assembly of coordinately activated transcription factors on the IFN-beta enhancer in vivo. Mol Cell. 1998;1(4):507-518.

87. Dugan JW, Albor A, David L, et al. Nucleotide oligomerization domain-2 interacts with $2^{\prime}-5^{\prime}$-oligoadenylate synthetase type 2 and enhances RNase-L function in THP-1 cells. Mol Immunol. 2009;47(2-3):560-566.

88. Sorbara MT, Ellison LK, Ramjeet M, et al. The protein ATG16L1 suppresses inflammatory cytokines induced by the intracellular sensors Nod1 and Nod2 in an autophagy-independent manner. Immunity. 2013;39(5):858-873.

89. Negroni A, Colantoni E, Vitali R, et al. NOD2 induces autophagy to control AIEC bacteria infectiveness in intestinal epithelial cells. Inflamm Res. 2016;65(10):803-813.

90. Müzes G, Tulassay Z, Sipos F. Interplay of autophagy and innate immunity in Crohn's disease: a key immunobiologic feature. World J Gastroenterol. 2013;19(28):4447-4454.

91. Homer CR, Richmond AL, Rebert NA, Achkar JP, McDonald C. ATG16L1 and NOD2 interact in an autophagy-dependent antibacterial pathway implicated in Crohn's disease pathogenesis. Gastroenterology. 2010;139(5):1630-1641.

92. Cadwell K. Crohn's disease susceptibility gene interactions, a NOD to the newcomer ATG16L1. Gastroenterology. 2010;139(5):1448-1450.

93. Salem M, Ammitzboell M, Nys K, Seidelin JB, Nielsen OH. ATG16L1: a multifunctional susceptibility factor in Crohn disease. Autophagy. 2015;11(4):585-594. 
94. Nguyen HT, Lapaquette P, Bringer MA, Darfeuille-Michaud A. Autophagy and Crohn's disease. J Innate Immun. 2013;5(5):434-443.

95. Cooney R, Baker J, Brain O, et al. NOD2 stimulation induces autophagy in dendritic cells influencing bacterial handling and antigen presentation. Nat Med. 2010;16(1):90-97.

96. Anand PK, Tait SW, Lamkanfi M, et al. TLR2 and RIP2 pathways mediate autophagy of Listeria monocytogenes via extracellular signal-regulated kinase (ERK) activation. J Biol Chem. 2011; 286(50):42981-42991.

97. Homer CR, Kabi A, Marina-García N, et al. A dual role for receptorinteracting protein kinase 2 (RIP2) kinase activity in nucleotidebinding oligomerization domain 2 (NOD2)-dependent autophagy. $J$ Biol Chem. 2012;287(30):25565-25576.

98. Urano F, Wang X, Bertolotti A, et al. Coupling of stress in the ER to activation of JNK protein kinases by transmembrane protein kinase IRE1. Science. 2000;287(5453):664-666.

99. Byndloss MX, Keestra-Gounder AM, Bäumler AJ, Tsolis RM. NOD1 and NOD2: new functions linking endoplasmic reticulum stress and inflammation. DNA Cell Biol. 2016;35(7):311-313.

100. Keestra-Gounder AM, Byndloss MX, Seyffert N, et al. NOD1 and NOD2 signalling links ER stress with inflammation. Nature. 2016;532(7599):394-397.

101. Caruso R, Núñez G. Innate immunity: ER stress recruits NOD1 and NOD2 for delivery of inflammation. Curr Biol. 2016;26(12): R508-R511.

102. Kaser A, Blumberg RS. Endoplasmic reticulum stress and intestinal inflammation. Mucosal Immunol. 2010;3(1):11-16.

103. Hosomi S, Kaser A, Blumberg RS. Role of endoplasmic reticulum stress and autophagy as interlinking pathways in the pathogenesis of inflammatory bowel disease. Curr Opin Gastroenterol. 2015;31(1):81-88.

104. Adolph TE, Tomczak MF, Niederreiter L, et al. Paneth cells as a site of origin for intestinal inflammation. Nature. 2013;503(7475):272-276.

105. Fritz T, Niederreiter L, Adolph T, Blumberg RS, Kaser A. Crohn's disease: NOD2, autophagy and ER stress converge. Gut. 2011;60(11):1580-1588.

106. Hoefkens E, Nys K, John JM, et al. Genetic association and functional role of Crohn disease risk alleles involved in microbial sensing, autophagy, and endoplasmic reticulum (ER) stress. Autophagy. 2013;9(12):2046-2055

107. Kaser A, Lee AH, Franke A, et al. XBP1 links ER stress to intestinal inflammation and confers genetic risk for human inflammatory bowel disease. Cell. 2008;134(5):743-756.

108. Montane J, Cadavez L, Novials A. Stress and the inflammatory process: a major cause of pancreatic cell death in type 2 diabetes. Diabetes Metab Syndr Obes. 2014;7:25-34.

109. Kim DH, Cheon JH. Pathogenesis of inflammatory bowel disease and recent advances in biologic therapies. Immune Netw. 2017;17(1): $25-40$.

110. Jostins L, Ripke S, Weersma RK, et al. Host-microbe interactions have shaped the genetic architecture of inflammatory bowel disease. Nature. 2012;491(7422):119-24.

111. Kim YG, Shaw MH, Warner N, et al. Cutting edge: Crohn's diseaseassociated Nod2 mutation limits production of proinflammatory cytokines to protect the host from Enterococcus faecalis induced lethality. J Immunol. 2011;187(6):2849-2852.

112. Salem M, Seidelin JB, Eickhardt S, Alhede M, Rogler G, Nielsen $\mathrm{OH}$. Species-specific engagement of human nucleotide oligomerization domain 2 (NOD)2 and Toll-like receptor (TLR) signalling upon intracellular bacterial infection: role of Crohn's associated NOD2 gene variants. Clin Exp Immunol. 2015;179(3):426-434.

113. Hugot JP, Zaccaria I, Cavanaugh J, et al; IBD International Genetics Consortium. Prevalence of CARD15/NOD2 mutations in Caucasian healthy people. Am J Gastroenterol. 2007;102(6):1259-1267.

114. Pauleau AL, Murray PJ. Role of nod 2 in the response of macrophages to toll-like receptor agonists. Mol Cell Biol. 2003;23(21):7531-7539.
115. Amendola A, Butera A, Sanchez M, Strober W, Boirivant M. Nod2 deficiency is associated with an increased mucosal immune regulatory response to commensal microorganisms. Mucosal Immunol. 2014;7(2):391-404.

116. Lesage S, Zouali H, Cézard JP, et al. CARD15/NOD2 mutational analysis and genotype-phenotype correlation in 612 patients with inflammatory bowel disease. Am J Hum Genet. 2002;70(4): 845-857.

117. Hampe J, Grebe J, Nikolaus S, et al. Association of NOD2 (CARD 15) genotype with clinical course of Crohn's disease: a cohort study. Lancet. 2002;359(9318):1661-1665.

118. Wehkamp J, Harder J, Weichenthal M, et al. NOD2 (CARD15) mutations in Crohn's disease are associated with diminished mucosal a-defensin expression. Gut. 2004;53(11):1658-1664.

119. Wehkamp J, Salzman NH, Porter E, et al. Reduced Paneth cell a-defensins in ileal Crohn's disease. Proc Natl Acad Sci U S A. 2005;102(50):18129-18134.

120. Simms LA, Doecke JD, Walsh MD, Huang N, Fowler EV, RadfordSmith GL. Reduced a-defensin expression is associated with inflammation and not NOD2 mutation status in ileal Crohn's disease. Gut. 2008;57(7):903-110.

121. Cadwell K, Liu JY, Brown SL, et al. A key role for autophagy and the autophagy gene Atg1611 in mouse and human intestinal Paneth cells. Nature. 2008;456(7219):259-263.

122. Wolfkamp SC, Verseyden C, Vogels EW, et al. ATG16L1 and NOD2 polymorphisms enhance phagocytosis in monocytes of Crohn's disease patients. World J Gastroenterol. 2014;20(10):2664-2672.

123. Tromp G, Kuivaniemi H, Raphael S, et al. Genetic linkage of familial granulomatous inflammatory arthritis, skin rash, and uveitis to chromosome 16. Am J Hum Genet. 1996;59(5):1097-1107.

124. Blau EB. Familial granulomatous arthritis, iritis, and rash. J Pediatr. 1985;107(5):689-693.

125. Wouters CH, Maes A, Foley KP, Bertin J, Rose CD. Blau syndrome, the prototypic auto-inflammatory granulomatous disease. Pediatr Rheumatol Online J. 2014;12:33.

126. Hasler P. Gabay C. Rheumatoid arthritis: from basic findings and clinical manifestations to future therapies. Semin Immunopathol. 2017;39(4):339-341.

127. Kim HW, Kwon YJ, Park BW, Song JJ, Park YB, Park MC. Differential expressions of NOD-like receptors and their associations with inflammatory responses in rheumatoid arthritis. Clin Exp Rheumatol. 2017;35(4):630-637.

128. Yuan H, Zelkha S, Burkatovskaya M, Gupte R, Leeman SE, Amar S. Pivotal role of NOD2 in inflammatory processes affecting atherosclerosis and periodontal bone loss. Proc Natl Acad Sci U S A . 2013;110(52):E5059-E5068

129. Liu HQ, Zhang XY, Edfeldt K, et al. NOD2-mediated innate immune signaling regulates the eicosanoids in atherosclerosis. Arterioscler Thromb Vasc Biol. 2013;33(9):2193-2201.

130. Johansson ME, Zhang XY, Edfeldt K, et al. Innate immune receptor NOD2 promotes vascular inflammation and formation of lipidrich necrotic cores in hypercholesterolemic mice, Eur J Immunol. 2014;44(10):3081-3092.

131. Zeidan MJ, Saadoun D, Garrido M, Klatzmann D, Six A, Cacoub P. Behçet's disease physiopathology: a contemporary review. Auto Immun Highlights. 2016;7(1):4.

132. Hamzaoui K, Abid H, Berraies A, Ammar J, Hamzaoui A. NOD2 is highly expressed in Behcet disease with pulmonary manifestations. J Inflamm (Lond). 2012;9(1):3.

133. Liu Y, Yang H, Liu LX, et al. NOD2 contributes to myocardial ischemia/reperfusion injury by regulating cardiomyocyte apoptosis and inflammation. Life Sci. 2016;149:10-17.

134. Nagata E, Oho T. Invasive Streptococcus mutans induces inflammatory cytokine production in human aortic endothelial cells via regulation of intracellular toll-like receptor 2 and nucleotide-binding oligomerization domain 2. Mol Oral Microbiol. 2017;32(2):131-141. 
135. Yao Q, Su LC, Tomecki KJ, Zhou L, Jayakar B, Shen B. Dermatitis as a characteristic phenotype of a new autoinflammatory disease associated with NOD2 mutations. J Am Acad Dermatol. 2013;68(4):624-631.

136. Macaluso F, Nothnagel M, Parwez Q, et al. Polymorphisms in NACHT-LRR (NLR) genes in atopic dermatitis. Exp Dermatol. 2007;16(8):692-698.

137. Wong CK, Chu IM, Hon KL, Tsang MS, Lam CW. Aberrant expression of bacterial pattern recognition receptor NOD2 of basophils and microbicidal peptides in atopic dermatitis. Molecules. 2016; 21(4):471.

138. Jiao D, Wong CK, Qiu HN, et al. NOD2 and TLR2 ligands trigger the activation of basophils and eosinophils by interacting with dermal fibroblasts in atopic dermatitis-like skin inflammation. Cell $\mathrm{Mol}$ Immunol. 2016;13(4):535-550.

139. Rodriguez-Nunez I, Caluag T, Kirby K, Rudick CN, Dziarski R, Gupta D. Nod2 and Nod2-regulated microbiota protect BALB/c mice from diet-induced obesity and metabolic dysfunction. Sci Rep. 2017;7(1):548
140. Denou, E. Lolmède K, Garidou L, et al. Defective NOD2 peptidoglycan sensing promotes diet-induced inflammation, dysbiosis, and insulin resistance. EMBO Mol Med. 2015;7(3):259-274.

141. Prajapati B, Jena PK, Rajput P, Purandhar K, Seshadri S. Understanding and modulating the toll like receptors (TLRs) and NOD like receptors (NLRs) cross talk in type 2 diabetes. Curr Diabetes Rev. 2014;10(3):190-200.

142. Deng B, Ye Z, Li L, et al. Higher expression of NOD1 and NOD2 is associated with Vogt-Koyanagi-Harada (VKH) Syndrome but not Behcet's disease (BD). Curr Mol Med. 2016;16(4):424-435.

143. Branquinho D, Freire P, Sofia C. NOD2 mutations and colorectal cancer - Where do we stand? World J Gastrointest Surg. 2016;8(4):284-293.

144. Castaño-Rodríguez N, Kaakoush NO, Mitchell HM. Patternrecognition receptors and gastric cancer. Front Immunol. 2014;5:336.

145. Kutikhin AG. Role of NOD1/CARD4 and NOD2/CARD15 gene polymorphisms in cancer etiology. Hum Immunol. 2011;72(10):955-968.

146. Liu J, He C, Xu Q, Xing C, Yuan Y. NOD2 polymorphisms associated with cancer risk: a meta-analysis. PLoS One. 2014;9(2):e89340.
Journal of Inflammation Research

\section{Publish your work in this journal}

The Journal of Inflammation Research is an international, peer-reviewed open access journal that welcomes laboratory and clinical findings on the molecular basis, cell biology and pharmacology of inflammation including original research, reviews, symposium reports, hypothesis formation and commentaries on: acute/chronic inflammation; mediators of
Dovepress

inflammation; cellular processes; molecular mechanisms; pharmacology and novel anti-inflammatory drugs; clinical conditions involving inflammation. The manuscript management system is completely online and includes a very quick and fair peer-review system. Visit http://www.dove press.com/testimonials.php to read real quotes from published authors. 